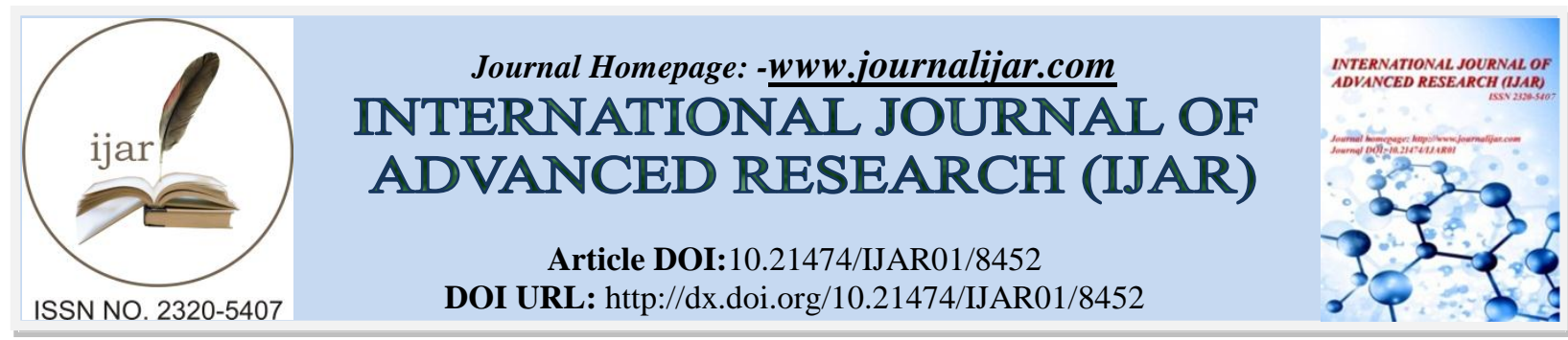

RESEARCH ARTICLE

\title{
CASE REPORT OF MASSIVE RECURRENT PERICARDIAL EFFUSION SECONDARY TO HYPOTHYROIDISM.
}

\author{
Y.Kettani, N.Azib, S. Hallab, L.Oukerraj and M. Cherti. \\ Cardiology B service, Ibn Sina hospital-Rabat. Morocco.
}

\section{Manuscript Info}

\section{Manuscript History}

Received: 20 November 2018

Final Accepted: 22 December 2018

Published: January 2019

\section{Key words:-}

hypothyroidism; pericardial effusion; cardiac tamponade; Echocardiography.

\section{Abstract}

Background:Although relatively rare, pericardial effusions secondary to hypothyroidism have been well described (1).

Moderate to large pericardial effusions are rare and associated tamponade is extremely rare (2). A wide variety of conditions can cause a pericardial effusion. Early recognition of a pericardial effusion and its underlying cause are important for improving prognosis and can avoid unnecessarily invasive investigations.

Case presentation: 62-year-old female patient without cardiovascular risk factors and treated for depression, admitted for the management of a state III dyspnea with severe asthenia Malaise and general weakness were also noted. The Cardiovascular Examination finds: Decreased Heart Sounds, with Signs of Right Heart Failure including Jugular Vein Turgescence with Hepatic jugular Reflux and Hepatomegaly, as well as lower limb edema reaching mid-leg. Pleuropulmonary examination found: $\mathrm{VM}$ decreased at baseline $\mathrm{SpO} 2=80 \%$. Abdominal examination found: Hepatomegaly. EKG: Sinuses and regular Rhythm at 80 BPM. And a diffuse Micro Voltage

Chest X-ray: shows cardiomegaly with a CTI> 0.6 and bilateral hilar overload with bilateral basithoracic pleural effusion. Transthoracic echocardiography was performed urgently at admission, which revealed circumferential pericardial effusion of great abundance.

The patient benefited the same day from a pericardial puncture with good clinical evolution.

The transthoracique echocardiography shows the persistence of low to moderate abundance of pericardial effusion, without pathological respiratory variations. No collapse of the VD-OD

The thoracoabdominopelvic CT scan shows the pericardial effusion and a sequential coronal renal notch.Biologically assessment: the patient has normochromic normocytic anemia with leucopenia (neutropenia) and hypothyroidism. The patient is transferred to cardiovascular chirurgical department for drainage and pleuropericardial window under medical treatment

Conclusion:Hypothyroidism should be ruled out in all patients with an unexplained pericardial effusion. The corollary is that, in hypothyroid patients, other more common causes of a pericardial effusion should be excluded. In patients with a large pericardial effusion due to

Corresponding Author:-Y.Kettani.

Address:-Cardiology B service, Ibn Sina hospital-Rabat. Morocco. 
hypothyroidism, cardiac tamponade may be present without significant tachycardia.

Copy Right, IJAR, 2017,. All rights reserved.

\section{Introduction:-}

Although relatively rare, pericardial effusions secondary to hypothyroidism have been well described (1). Moderate to large pericardial effusions are rare and associated tamponade is extremely rare (2). Most reports have emphasized the rarity of presentations involving cardiac tamponade, and few reports have discussed the difficulties of early diagnosis (2-5). A wide variety of conditions can cause a pericardial effusion. Early recognition of a pericardial effusion and its underlying cause are important for improving prognosis and can avoid unnecessarily invasive investigations.

\section{Observation:-}

She was a 62-year-old female patient without cardiovascular risk factors and treated for depression, admitted for the management of a state III dyspnea with severe asthenia Malaise and general weakness were also noted. The progression was marked by the exacerbation of her dyspnea with orthopnea, Nocturnal Paroxystic Dyspnea and increased volume of the lower limbs, which prompted her consultation two months ago with a cardiologist who discovered a circumferential pericardial effusion of great abundance and referred it to the emergency department, where she benefited from an evacuating pericardial puncture and a pleural puncture. The evolution is marked by the reappearance of dyspnea state III of the NYHA with sensation of chest tightness.

Clinical examination found: a conscious patient, well oriented in time and space, eupneic at rest, cannot support Dorsal Decubitus, TA: 130/80 mmHg, RC: 75 BPM, discomposed conjunctiva, digital Hippocratism. The Cardiovascular Examination finds: Decreased Heart Sounds, with Signs of Right Heart Failure including Jugular Vein Turgescence with Hepatic jugular Reflux and Hepatomegaly, as well as lower limb edema reaching mid-leg. Pleuropulmonary examination found: VM decreased at baseline SpO2 $=80 \%$. Abdominal examination found: Hepatomegaly. Examination of the thyroid: free. The rest of the exam is peculiar. EKG: Sinuses and regular Rhythm at 80 BPM. And a diffuse Micro Voltage

Chest X-ray: shows cardiomegaly with a CTI> 0.6 and bilateral hilar overload with bilateral basithoracic pleural effusion.

Fig. 1:-Chest X-ray showing CMG.

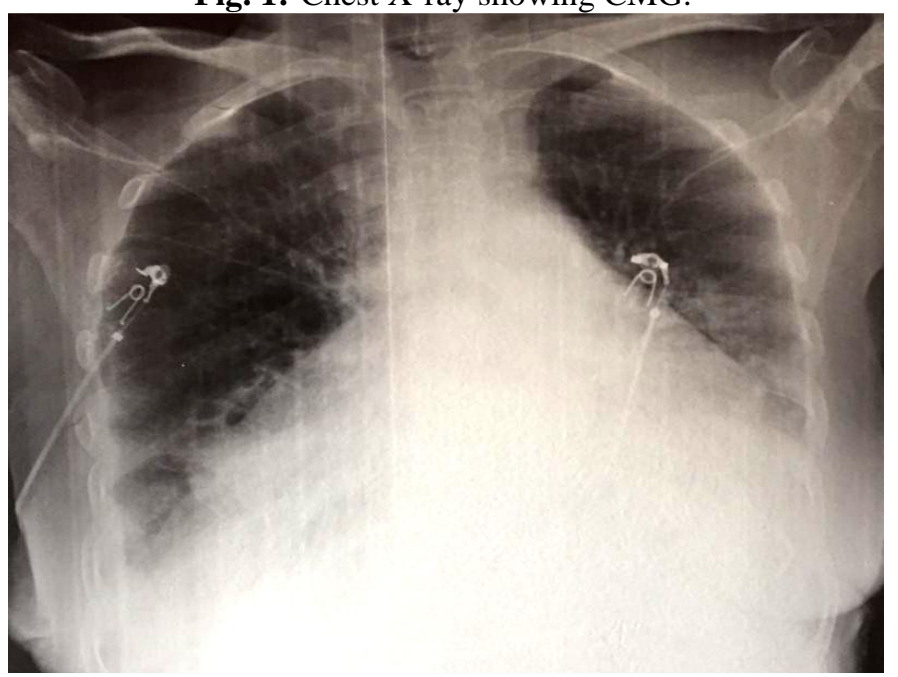

Transthoracic echocardiography was performed urgently at admission, which revealed circumferential pericardial effusion of great abundance. 
Fig. 2:-TTE showing circumferential pericardial effusion of great abundance.

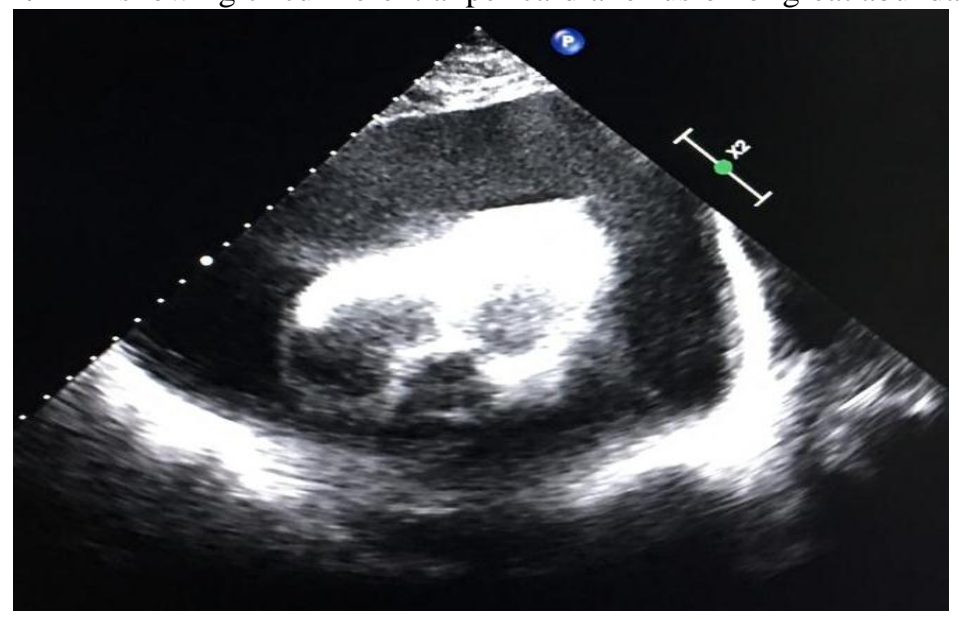

Evolution: The patient benefited the same day from a pericardial puncture with good clinical evolution.

The transthoracique echocardiography shows the persistence of low to moderate abundance of pericardial effusion, without pathological respiratory variations. No collapse of the VD-OD with: $4 \mathrm{~mm}$ of liquid next to the wall of the right ventricle, $43 \mathrm{~mm}$ next to the post wall of the left ventricle, $41 \mathrm{~mm}$ next to the side wall of the left ventricle, 12 $\mathrm{mm}$ next to Apex of the left ventricle, $17 \mathrm{~mm}$ in retro right atrium.

Fig 3, 4, 5:-transthoracique echocardiography shows the persistence of low to moderate abundance of pericardial

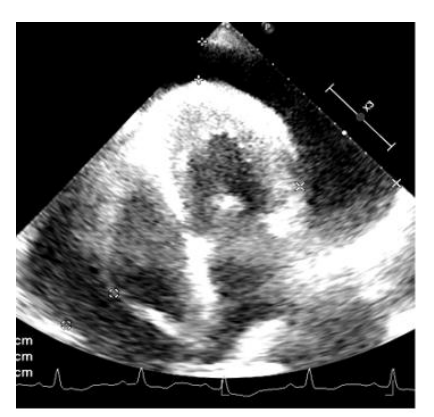
effusion.
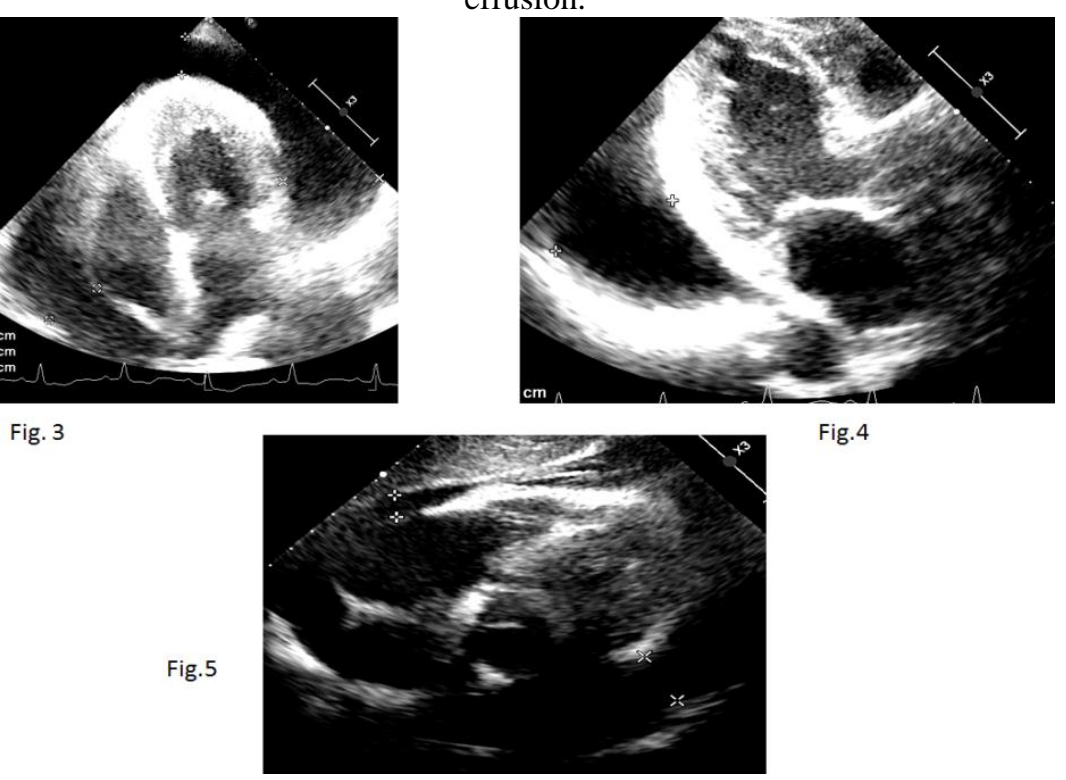

Fig.4

The thoracoabdominopelvic CT scan shows the pericardial effusion and a sequential coronal renal notch. 
Fig 6:-thoracoabdominopelvic CT scan shows the pericardial effusion and a sequential coronal renal notch.

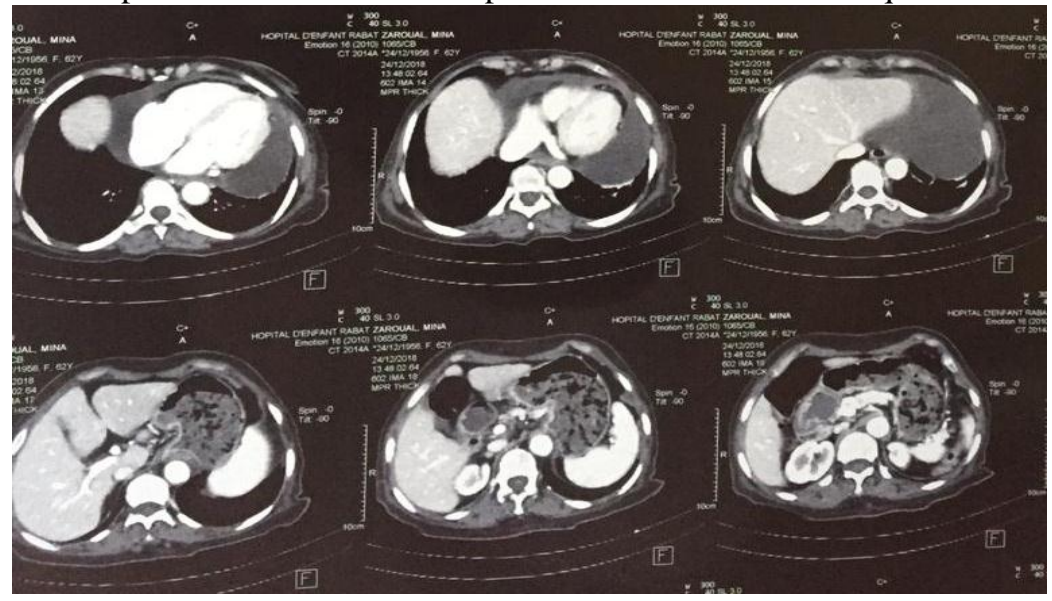

Biologically assessment: the patient has normochromic normocytic anemia with leucopenia (neutropenia) and hypothyroidism

Tab 1:-result of biological assessment realized.

\begin{tabular}{|l|l|l|}
\hline $\mathrm{Hb}=10.7 \mathrm{~g} / \mathrm{dl}$ & $\mathrm{Na}+=135 \mathrm{mEq} / 1$ & $\mathrm{CRP}=21 \mathrm{mg} / 1$ \\
$\mathrm{WBC}=3080 / \mu \mathrm{l}$ & $\mathrm{K}+=4.8 \mathrm{mEq} / 1$ & Troponin $=0.014$ \\
$\mathrm{Plq}=288000 / \mu \mathrm{l}$ & Urea $=0.33 \mathrm{~g} / 1$ & $\mathrm{TSH}=4.063$ \\
$\mathrm{TP} 3=1.40 \downarrow$ \\
$\mathrm{CA} 19.9=11.2$ & Creatinin $=7.4 \mathrm{mg} / 1$ & $\mathrm{~T} 4=0.50 \downarrow$ \\
$\mathrm{ACE}=10 \uparrow$ & $\mathrm{CA} 125=47.7 \uparrow$ & \\
& $\beta \mathrm{HCG}<1.20$ & Ferritin $=585 \uparrow$ \\
\end{tabular}

1. BK pericardial fluid: negative direct examination and negative culture.

2. PCR pericardial fluid: DNA - and Rifampicin resistance gene -

3. The patient is transferred to cardiovascular chirurgical department for drainage and pleuropericardial window under medical treatment including Aspirin and colchicines and thyroid hormone substitution (Levothyrox) and PPI

\section{Discussion:-}

The occurrence of a pericardial effusion in hypothyroidism seems to be related to the severity and duration of the disease. The incidence is reported to be as low as $3 \%$ in an early mild stage to $80 \%$ when myxedema is present (1, 6). In contrast to the frequent occurrence of small pericardial effusions in patients with hypothyroidism, moderate to large pericardial effusions are rare and associated tamponade is extremely rare. Cardiac tamponade in patients diagnosed with hypothyroidism is probably as rare as it is due to pericardial distensability and the slow accumulation of fluid, allowing significant fluid accumulation without hemodynamic compromise (2). A wide variety of medical conditions, including malignancy, can lead to a pericardial effusion. In approximately $45 \%$ of cancer patients with symptomatic pericardial disease, malignancy is not directly to blame (7). Malignancy is also a cause of hypothyroidism, and hypothyroidism is also a well-documented complication after head and neck cancer treatment.

The presumptive mechanism underlying exudative pericardial effusions is extravasation of hygroscopic mucopolysaccharides into the pericardial space along with increased capillary permeability, decreased lymphatic drainage, and increased retention of salt and water (2). Cold exposure is one of the precipitants of myxedema, and exposure to cold has been reported to induce declines in serum T3 and T4, and to increase T3 clearance and production rates $(9)$.

Lin et al. reported a case of myxedema associated with cardiac tamponade that occurred in the winter, and it is conceivable that circannual changes in thyroid hormone physiology also may have contributed to the clinical presentation in this patient (5). 
Several points arising from this case are worth highlighting. First, diagnosis of hypothyroidism in the ED is difficult, because hypothyroidism in adults classically has an insidious onset with a range of non-specific symptoms that can delay the diagnosis by months or even years. The clinical features of hypothyroidism, such as weight gain, fatigue, cold intolerance, constipation, dry skin, edema, and slow mentation are usually subtle and may be overlooked. The diagnosis is rarely made on clinical grounds alone, particularly in the elderly, who are sometimes confused with either depression or early dementia $(3,4,10$, and 11$)$. Due to its insidious onset, a pericardial effusion secondary to hypothyroidism frequently occurs without hemodynamic change, and is apparent only on echocardiography (4). Such findings in an asymptomatic elderly patient can be misleading and may lead to unnecessarily invasive procedures such as pericardiocentesis (4).

Secondly, an early ED ultrasound should be performed to establish the presence of a pericardial effusion. Unexplained hypotension or dyspnea, enlarged cardiac silhouette, pericarditis, and chest pain or dyspnea with underlying disease, such as cancer, uremia, or systemic lupus erythematosis, are high-risk populations for pericardial effusions. Using bedside echocardiography, cardiologic physician can detect a pericardial effusion with a sensitivity of $96 \%$, specificity of $98 \%$, and overall accuracy of $97.5 \%$ (12). Thirdly, identification of clinical signs suggestive of hypothyroidism and obtaining thyroid function tests in the ED in appropriate patients are crucial to an early diagnosis. When an EP is presented with a patient with a moderate to large pericardial effusion, the challenge is to identify its etiology. Sagrista-Sauleda et al., in a prospective study of 322 patients, discussed simple clinical clues such as underlying disease, the presence or absence of inflammatory signs, and the development of tamponade that are useful for establishing an etiology (13). In the majority of patients, the cause of effusion can be presumed to be related to an underlying condition (13). A detailed review of the history, complete physical examination and appropriate noninvasive studies have a diagnostic yield of 55\% (13). Due to its invasiveness and low diagnostic yield (7\%), pericardiocentesis is indicated only in patients with overt clinical tamponade, in patients suspected of having a purulent Pericarditis, and in patients with an idiopathic, chronic large pericardial effusion (14). Hypothyroidism is relatively rare $(1.5 \%)$ but remains a significant cause of moderate to severe pericardial effusions (13). A hemodynamically insignificant pericardial effusion and unremarkable clinical symptoms are the most characteristic features of hypothyroidism.

Nevertheless, screening is still useful for identifying patients with overt but clinically unrecognized thyroid disease (11). A non-selective screening test with a sensitive thyroid-stimulating hormone for thyroid dysfunction, broadly applied, has been found to be more effective than clinical judgment, indeed, the eventual diagnosis of hypothyroidism was made only after routine investigations in the majority of case reports $(2-4,11)$. Accordingly, hypothyroidism must be ruled out in all patients with an unexplained pericardial effusion, not just in patients with clinically obvious hypothyroidism or the elderly (3). Finally, once the diagnosis has been established, treatment with thyroid replacement hormones usually leads to the resolution of the effusion over 2-12 months without sequel (15).

By the way, Thyroid gland also has a crucial effect on erythropoiesis by induction of erythropoietin secretion and also proliferation of erythroid progenitors, the most common thyroid dysfunctions, hypothyroidism and hyperthyroidism affect blood cells and cause anemia with different severity. These thyroid disorders also cause thrombocytopenia, leucopenia and even in rare cases cause pancytopenia (in hypothyroidism). (8).

\section{Conclusion:-}

The clinical features of hypothyroidism can be subtle and the diagnosis is often delayed. A haemodynamically insignificant pericardial effusion and unremarkable clinical symptoms are not infrequently seen in this setting. Therefore, hypothyroidism should be ruled out in all patients with an unexplained pericardial effusion. The corollary is that, in hypothyroid patients, other more common causes of a pericardial effusion should be excluded. In patients with a large pericardial effusion due to hypothyroidism cardiac tamponade may be present without significant tachycardia.

\section{References:-}

1. Hardisty CA, Naik DR, Munro DS. Pericardial effusion in hypothyroidism. Clin Endocrinol 1980;13:359-64.

2. Manolis AS, Varriale P, Ostrowski RM. Hypothyroid cardiac tamponade. Arch Intern Med 1987;147:1167-9.

3. Gupta R, Munyak J, Haydock T, Gernsheimer J. Hypothyroidism presenting as acute cardiac tamponade with viral pericarditis. Am J Emerg Med 1999;17:176-8. 
4. Zimmerman J, Yahalom J, Bar-On H. Clinical spectrum of pericardial effusion as the presenting feature of hypothyroidism. Am Heart J 1983;106:770 -1.

5. Lin CT, Liu CJ, Lin TK, Chen CW, Chen BC, Lin CL. Myxedema associated with cardiac tamponase. Jpn Heart J 2003;44:447-50.

6. Kabadi UM, Kumar SP. Pericardial effusion in primary hypothyroidism. Am Heart J 1990;120:1393-5.

7. Wilkes JD, Fidias P, Vaickus L, Perez RP. Malignancy-related pericardial effusion. 127 cases from the Roswell Park Cancer Institute. Cancer 1995;78:1377- 87.

8. Kawa MP, Grymuła K, Paczkowska E, Baśkiewicz-Masiuk M, Dąbkowska E, Koziołek M, et al. Clinical relevance of thyroid dysfunction in human haematopoiesis: biochemical and molecular studies. Eur $\mathrm{J}$ Endocrinol. 2010;162(2):295-305. [PubMed]

9. Reed HL. Circannual changes in thyroid hormone physiology: the role of cold environmental temperatures. Arctic Med Res 1995; 54(Suppl 2):9 -15.

10. Lindsay RS, Toft AD. Hypothyroidism. Lancet 1997;349:413-7.

11. Helfand M, Redfern CC. Clinical guideline, Part 2. Screening for thyroid disease: an update. Ann Intern Med 1998;129:144-58.

12. Mandavia DP, Hoffner RJ, Mahaney K, Henderson SO. Bedside echocardiography by emergency physicians. Ann Emerg Med 2001;38:377- 82.

13. Sagrista-Sauleda J, Merce J, Permanyer-Miralda G, Soler-Soler J. Clinical clues to the causes of large pericardial effusion. Am J Med 2000;109:95-101.

14. Soler-Soler J, Sagrista-Sauleda J, Permanyer-Miralda G. Management of pericardial effusion. Heart 2001;86:235- 40 .

15. Khaleeli AA, Memon N. Factors affecting resolution of pericardial

16. effusions in primary hypothyroidism: a clinical, biochemical and echocardiographic study. Postgrad Med J 1982;58:473- 6 . 